\section{ANNOUNCEMENT}

\section{AMS Short Course on Integrating NWP System Components Using Container Technology, 5 January 2019, Phoenix, Arizona}

Software systems require substantial setup to get all the necessary code, including external libraries, compiled on a specific platform. Recently, the concept of containers has been gaining popularity because they allow for software systems to be bundled (including operating system, libraries, code, and executables) and provided directly to users, eliminating possible frustrations with up-front system setup. This course will provide information on using software containers that have been established for community use to quickly spin up a numerical weather prediction (NWP) forecast system [using the Weather Research and Forecasting Model (WRF) initialized with WRF Pre-processing System (WPS) and the Gridpoint Statistical Interpolation (GSI) data assimilation system] that can then be post-processed [using the Unified Post Processor (UPP)] and verified [using the Model Evaluation Tools (MET)]. Ultimately, the established containers substantially reduce the spin-up time with setting up and compiling software systems and promote greater efficiency in getting to the end goal of producing model output and statistical analyses. The goal of this course is to raise awareness about tools and facilities available to the community for testing and evaluating of NWP innovations, including the emerging set of software tools in reusable containers.

While this course may appeal to a wide-reaching audience, this information may be particularly useful to undergraduate and graduate students interested in learning more about NWP and to university faculty that may find software containers to be a useful teaching tool to add to their course curriculum. In addition, researchers who wish to collaborate with others may find these tools useful for sharing a code base and replicating procedures even if they are not working on the same platform.

The format of the course will consist of a general overview of the NWP system components available in Docker containers (e.g., WPS/WRF, GSI, UPP, MET, METViewer) followed by the opportunity for participants to conduct hands-on learning exercises by running an end-to-end NWP system through specific usage examples with Docker containers.
Requirements are as follows:

- laptop (Mac or Windows) with a recent operating system that supports Docker, such as RHEL7 or Windows10 (visit https://www .docker.com to download and install the version compatible with your system);

- basic UNIX proficiency; and

- introductory knowledge of numerical weather prediction.

The instructors for the course include John Halley Gotway, Tara Jensen, Michael Kavulich Jr., and Jamie Wolff (National Center for Atmospheric Research, Boulder, Colorado). For more information, please contact Jamie Wolff (National Center for Atmospheric Research, 3450 Mitchell Ln., Boulder, CO 80301; tel: 303-497-2812; email: jwolff@ucar .edu). (10/18)

\section{ANNOUNCEMENT}

\section{AMS Short Course on Tutorial on How to Build a 3D-Printed Auto- matic Weather Station, 5 January 2019, Phoenix, Arizona}

Accurate and reliable real-time monitoring and dissemination of observations of atmospheric and

\title{
2019 AMS ANNUAL MEETING CALL FOR PAPERS
}

\section{6-10 January 2019, Phoenix, Arizona}

The 2019 AMS Annual Meeting theme is "Understanding and building resilience to extreme events by being interdisciplinary, international, and inclusive (III)." For the full description of the theme, please visit the AMS 2019 Annual Meeting website at https://annual.ametsoc.org/. Preliminary programs, registration, hotel, and general information will be posted on that website in late September 2018.

The deadline for abstracts has passed. Authors of accepted presentations will be notified via e-mail by lateSeptember 2018.

All extended abstracts are to be submitted electronically and will be available online. Instructions for formatting extended abstracts will be posted on the AMS website. Authors have the option to submit extended abstracts (up to $10 \mathrm{MB}$ ) electronically by 15 February 2019. All abstracts, extended abstracts, and presentations will be available on the AMS website at no cost. 
hydrologic conditions in general is critical for a variety of research and decision support applications. The University Corporation for Atmospheric Research (UCAR), with support from USAID, has started an initiative to develop low-cost automatic instrumentation including temperature, pressure, relative humidity, light, wind speed, wind direction, and tipping bucket sensors. One important aspect of this initiative is to make the designs and documentation open source to the community. The instrumentation have been developed using innovative new technologies such as $3 \mathrm{D}$ printers, Raspberry Pi computing systems, and wireless communications.

The goal of this short-course is to provide participants with hands-on experience to build sensor components of a 3D printed weather station using 3D printer technology, microsensors, and single board computers. The course will provide participants experience using 3D printers to print, assemble, and test sensors (anemometer, wind vane, etc.). Over the oneday course, the participants will gain experience using the open-source Python sensor code and sending data to the cloud. After the course, the participants should have knowledge to build the other sensors using the 3D-Printed Automatic Weather Station (3D-PAWS) open source project that has been developed to support education, outreach, and capacity building.

The course will be divided into two parts. The first part will be a series of presentations providing an overview of the 3D-PAWS project: the printing, assembling, software, and installation processes. The second part will be a hands-on demonstration of building a 3DPAWS sensor. During this part of the course, participants will have an opportunity to work with the $3 \mathrm{D}$ printers, assembling sensors, using the Python software to make measurements. Stations will be setup in the classroom to provide participants experience working with the different components of the $3 \mathrm{D}$ printing and assembling process.

The course format consists of one day of lectures followed by two hours of hands on laboratory session with exercises that can be completed any time during the conference. The instructors for the course will be Paul A. Kucera and Martin Steinson (UCAR, Boulder, Colorado). Computers, laptops or internet access will not be available or required for this course.

For more information, please contact Paul Kucera (UCAR, P.O. Box 3000, Boulder, CO 80307; tel: 303-497-2807; email: pkucera@ucar .edu). $(10 / 18)$

\section{ANNOUNCEMENT}

AMS Short Course on Python for Synoptic Meteorology Using MetPy, 6 January 2019, Phoenix, Arizona

The use of the Python programming language has grown immensely over the past decade and has become an essential tool within education, research, and industry within the atmospheric sciences. This course aims to go beyond a basic Python introduction and help attendees advance their ability to apply Python to practical problems in meteorology through hands-on exercises. This includes topics such as remote data access, calculation of derived quantities, and plotting of these quantities on map projections. As a more intermediate workshop, this workshop assumes a basic knowledge of Python syntax and some familiarity with scientific Python libraries like NumPy and Matplotlib. The goal of the course is to have attendees learn how to apply Python to practical meteorology problems through use of the MetPy library. They will gain experience accessing remote datasets, using MetPy to calculate derived quantities, and plotting these quantities on weather maps, including station plots. Synoptic meteorology serves as a backdrop for these activities, with motivating examples for case studies such as visualization of satellite imagery, quasigeostrophic/ isentropic analysis, and soundings. This course is extensively hands-on through the use of Jupyter notebooks and will consist of one day of interactive lecture sessions with incorporated exercises that will be completed during the short course. In addition, the afternoon sessions will be aimed at developing Jupyter notebooks that will launch each attendee to bring something tangible home from the course.

The instructors for the course are Dr. Ryan May (UCAR/Unidata), Dr. John Leeman (UCAR/Unidata), and Dr. Kevin Goebbert (Valparaiso University). Internet access will be available for this course. Computers and laptops will not be available but are required for this course. Windows, MacOS, and Linux operating systems are generally supported. Participants will be emailed installation instructions for the course Python environment in advance of the course.

For more information, please contact Ryan May (tel: 303-497-8679; email: rmay@ucar.edu).(10/18)

\section{ANNOUNCEMENT}

\section{AMS Short Course on An Introduc- tion to Using the NASA Giovanni System for Multidisciplinary Re- search and Applications, 6 January 2019, Phoenix, Arizona}

Giovanni is one of the pioneering web services provided to the public for the analysis of NASA Earth science data. Beginning in 2003, Giovanni has given users-both 
neophytes and experts-a way to explore remote sensing and model data with minimal investment in time and software. As it has grown and evolved, Giovanni has added many more analytical capabilities and has markedly improved system performance and speed. It now features a large and varied library of data products providing information about weather, climate, atmospheric composition and dynamics, oceanography, hydrological processes, and Earth system interactions. Scientists and educators frequently note Giovanni's ease of use, which provides both a way to find data of interest for a wide variety of research areas, and it serves as an introductory scientific research environment useful for either guided or independent investigations.

Participants in this short course will 1) learn how to use Giovanni; 2) learn about the NASA Goddard Earth Sciences Data and Information Services Center's (GES DISC) large collection of NASA satellite and modeling data products, including global precipitation, atmospheric composition, atmospheric dynamics, hydrology, and solar irradiance; 3 ) conduct several hands-on data visualization scenarios demonstrating Giovanni capabilities; 4) learn how to use Giovanni to create multidisciplinary data analyses; and 5) provide comments and feedback for future refinements of Giovanni.

Giovanni has recently expanded its capabilities regarding the usage of datasets from multiple disciplines, particularly those related to meteorology and hydrology. This short course will provide focused insight on those capabilities and those datasets.

The morning session of the short course will provide an introduction to the NASA GES DISC, a preliminary description of Giovanni, and a tour of the Giovanni interface and system architecture. This will be followed by a summary of the major Giovanni data holdings relevant to the meteorological community and the first of two sessions on Giovanni features. After lunch (not provided), the second session on Giovanni features will precede an extended period of hands-on exercises conducted with GES DISC staff supervision and assistance. By the end of the one-day short course, participants will possess the necessary knowledge and skills to perform multidisciplinary research with Giovanni.

Participants should bring their own laptop computer so that they can participate and benefit fully from the hands-on exercises during the course. Internet access will be provided.

For more information please contact James Acker (NASA GSFC, Code 610.2, Greenbelt, MD 20771; tel.: 301-614-5435; email: james.g.acker @nasa.gov) or Zhong Liu (NASA GSFC, Code 610.2, Greenbelt, MD 20771; tel.: 301-614-5764; email: zhong.liu-1@nasa.gov). (10/18)

\section{ANNOUNCEMENT}

\section{AMS Short Course on Applying JPSS Data Products to Better Fore- cast Challenging Weather Events, 6 January 2019, Phoenix, Arizona}

Please join us for a short course on applying the new generation Joint Polar Satellite System (JPSS) capabilities to a variety of operational forecasting scenarios. Participants will have an opportunity for handson experiences using Suomi National Polar-Orbiting Partnership (NPP) and NOAA-20 (JPSS-1) data to address challenging operational weather scenarios. The course will provide information on JPSS instruments and their improved observational capabilities, demonstrate the operational application of the new JPSS products using recent weather scenarios and the methods available for accessing those data products, and discuss online resources available for training. The demonstration exercises will also show how having two polarorbiting satellites (S-NPP and NOAA20) separated by 50 minutes provides additional critical data.

The goal of the course is to make users of polar-orbiting environmental satellite data aware of JPSS capabilities and how to apply those capabilities to improve environmental observation and forecasting. The course will benefit all current operational forecasters, researchers, and broadcasters. It is anticipated that students, university faculty, nonmeteorologists, and the general public will find this course very interesting. The course format will consist of a balanced mix of presentations and hands-on exercises demonstrating the applications for the current JPSS data products and new capabilities under development for operational applications.

Instructors incluce Mitch Goldberg (NOAA/NESDIS); Arron Layns (NESDIS/JPSS); Chris Barnet and Bonnie Reed (Science and Technology Corporation); Steven Miller and Jorel Torres (CIRA); Eric Stevens (GINA); Dan Nietfeld (NOAA/ NWS); Kathleen Strabala (CIMSS); Lihang Zhou, Shoba Kondagrunta and Ivan Csiszar (NESDIS/STARCollege Park); Michael Bowlan (NWS/SPC); Michael Folmer (NWS/ OPC); Michael Pavolonis (NESDIS/ STAR-Madison); Douglas Wesley and Jeffrey Osiensky (NWS/AAWU); Carl Dierking (University of Alaska); Andy Edman (NWS/WR); Dan Nietfeld and Ravan Ahmadov (OAR/ ESRL); and Evan Ellicott (University of Maryland).

A luncheon will be provided during the short course. Participants are encouraged to bring their laptops or tablets for the hands-on exercises. For more information, please contact Gary McWilliams (email: gary .mcwilliams@noaa.gov).(10/18) 
ANNOUNCEMENT

AMS Short Course on GOES-R Series: Forecasting Applications, 6 January 2019, Phoenix, Arizona

NOAA's Geostationary Operational Environmental Satellite R Series (GOES-R) now has two satellites in orbit and we are continually learning more about the capabilities of these next generation satellites. The Geostationary Lightning Mapper (GLM) and 11 new channels on the 16-channel Advanced Baseline Imager (ABI) have substantially improved spatial resolution and temporal refresh rate. These new measurements produce more timely, detailed, and accurate information than ever before. Short-course participants will have the opportunity to receive hands-on experience with GOES-16 data, showcasing the many applications to help improve forecasts and warnings of high impact weather and environmental phenomena.

The primary goal of this course is to familiarize users with GOES-R capabilities and how it can improve their services to customers, saving lives and property. The course will showcase a brief overview of the ABI's 16 channels, GLM, insight on how to access existing resources (web page, educational pages, etc.), and an understanding of the options available to acquire GOES-R imagery and products. The course will then immerse students into three different forecasting challenge topics: aviation forecasting, fire weather, and convective weather. Each of these topics will cover specific GOES-R products and applications with hands-on case studies and lab exercises. The overarching goal is to introduce users to techniques and applications to improve observations, forecasting, and warnings to help bridge the gap between old and new in the most efficient manner in a field where every minute counts.

Instructors include subject matter experts from NOAA/NESDIS and various cooperative institutes. Participants are expected to bring their own laptops to be able to participate in the hands-on exercises.

For more information please contact Janel Thomas (email: janel.thomas @noaa.gov). (10/18)

\section{ANNOUNCEMENT}

AMS Short Course on Machine Learning in Python for Environmental Science Problems, 6 January 2019, Phoenix, Arizona

Interest in artificial intelligence, machine learning, and deep learning in the environmental sciences has grown rapidly in conjunction with the increased presence of AI in our daily lives. Many people now want to apply machine learning to their own data and problems but do not know where to start. The goal of this course is to teach participants how to develop a machine-learning workflow, using open-source Python tools to solve common types of problems in the atmospheric, oceanic, and environmental sciences. Specifically, this short course will teach participants how to use Python machine-learning and deep-learning libraries to process a dataset, train different models, generate and evaluate predictions for previously unseen cases, and interpret models to gain physical understanding of what they have learned.

Participants will use a series of Jupyter notebooks to explore severe weather data, train various machinelearning models, and interact with Python's machine-learning pipeline with a focus on xarray, pandas, scikitlearn, and Tensorflow. The notebooks will be hosted on a cloud-computing service with access to graphical processing units (GPUs), which will enable participants to train and interpret deep learning models in real time.

All participants need to bring a laptop with a power adapter and the ability to connect wirelessly to the internet via the conference center network. Participants should have a basic understanding of the Python programming language, but no previous machine learning or data science experience is required. Additional instructions will be emailed to registered participants before the course begins.

The instructors for this course are David John Gagne II (National Center for Atmospheric Research, Boulder, Colorado), Sheri Mickelson (National Center for Atmospheric Research, Boulder, Colorado), Ryan Lagerquist (University of Oklahoma, Norman, Oklahoma), and Greg Herman (Colorado State University, Fort Collins, Colorado). A lunch will be provided during the short course.

For more information please contact David John Gagne ( National Center for Atmospheric Research, P.O. 3000, Boulder, CO 80307; tel.: 303-497-2714; email: dgagne@ucar .edu; Twitter: @DJGagneDos). (10/18)

\section{ANNOUNCEMENT}

AMS Short Course on Weather and Phased Radar Polarimetry, 6 January 2019, Phoenix, Arizona

Radar polarimetry with multiparameter measurements has matured to the point that it has been implemented on the national network of WSR-88D Doppler radars. While the technology of radar polarimetry has matured and polarimetric radar data (PRD) are available nationally and worldwide, radar polarimetry is still in its initial stages for operational usage. There is a lot of room for research and development, especially in using PRD. Phased array technology has recently been introduced to the weather community to increase data update rates to lengthen the lead time of weather hazard warnings. Polarimetric phased array radar is desirable for future weather 
observations and multimission capabilities.

This Sunday morning short course will provide the background information on weather radar polarimetry and polarimetric phased array radar (PPAR) and their applications and will introduce the latest advances in research and development of a PPAR that can serve the multiple functions (e.g., weather and aircraft surveillance). The course will be divided into three parts. The first will provide concepts, principles, and applications of weather radar polarimetry. The second part will discuss the reasons for polarimetric phased array radar (PPAR) for fast data update and recent advancements in developing a PPAR for multimission applications. The third part will give students hand-on experience in calculating polarimetric radar variables and processing polarimetric radar data using Matlab. The instructor for the course are Prof. Guifu Zhang (University of Oklahoma) and Prof. Richard J. Doviak (National Severe Storms Laboratory). A luncheon will be provided during the short course. Laptops will be required for this course. For more information please contact Guifu Zhang (OU/SoM., 120 David L. Boren Blvd., Suite 5900, Norman, OK 73072; tel: 405-325-3507; email: guzhang1 @ou.edu). (10/18)

\section{ANNOUNCEMENT}

AMS Short Course: A Beginner's Course to Using Python in Climate and Meteorology, 5-6 January 2019, Phoenix, Arizona

The application of object-oriented programming and other advances in computer science to the atmospheric sciences has in turn led to advances in modeling and analysis tools and methods. The open-source language Python has been at the forefront of the application of such advances, through general science packages such as scipy and matplotlib, as well as atmospheric science-specific projects such as PCMDI's UV-CDAT and ESG end-user tools and NCAR's PyNGL, resulting in a robust computing environment for all kinds of atmospheric science, including (but not limited to): modeling, time series analysis, air quality data analysis, satellite data processing, in situ data analysis, GIS, visualization, gridding, model intercomparison, workflow integration, and very large (terabyte) dataset manipulation and access.

Still, to many atmospheric scientists, object-oriented programming-Python in particular-seems mysterious and remote; as a result, they find the idea of learning Python to be daunting. Additionally, while a number of tutorials and other curricula exist to introduce a newcomer to Python, few are geared to the specific needs of atmospheric scientists. This course provides a gentle introduction to Python for the atmospheric scientist, specialized to the needs of the field. While we expect all participants will have basic programming experience-including basic knowledge of variables (integers, floats, strings), loops, conditionals (if/ then), and functions-no other exposure to Python or object-oriented programming is assumed. If you are a moderately experienced Python programmer, this course will be a poor fit for you.

All attendees will need to bring a laptop (with power adapter) that has the Enthought Canopy Python distribution installed on it. Windows, Mac OS X, and Linux laptops are all acceptable; tablets are discouraged. Instructions for doing the required installations are available in advance of the course (at http://www.johnny -lin.com/ams/begpy.shtml). Because the course is two days, to maximize learning value for students, there will be optional homework assigned at the end of day one that will be discussed the next day.
For more information please contact the instructor of the course, Dr. Johnny Lin, Computing and Software Systems Division, University of Washington Bothell, Bothell, Washington and the Physics and Engineering Department, North Park University, Chicago, Illinois (email: johnny@johnny-lin.com). (10/18)

\section{ANNOUNCEMENT}

AMS Short Course on Using NASA High Performance Computers to Perform Analytics on Large Earth Science Data, 6 January 2019, Phoenix, Arizona

Downloading large NASA Earth Science datasets is time consuming and requires large local storage resources. The NCCS has addressed this problem by repackaging Earth science data, including major atmospheric and oceanic reanalysis datasets and downscaled climate projection datasets. To provide access to scientists worldwide, we have developed visualization and analytics tools to utilize NCCS resources to explore the data. The visualization tool CREATE-V provides quick look access to the reanalyses data, while the Earth Data Analytics Service (EDAS) provides server-side analytics to reanalysis, climate model, and observational data. The course is aimed at students and scientists who are interested in utilizing high performance computing on NASA Earth Science data using the latest in server-side technology.

The course will be divided into two parts. The first will provide a description of the tools, including web visualization (CREATE-V), serverside analytics (EDAS) using Python and Jupyter notebooks, and selected GIS services available through an ArcGIS portal. The available data will be described and science examples (including subsetting and EOF analysis) will be provided. The second part will 
be a hands-on opportunity for participants to work through examples as well as developing their own code that will then run on NASA highperformance computing resources.

The course format consists of 1.5 hours of lecture followed by a twohour hands-on session with exercises that can be completed during or after the short course. The instructors for the course are associated with the NASA Center for Climate Simulation at the NASA Goddard Space Flight Center: Jerry Potter, Laura Carriere, Tom Maxwell, and Dan Duffy

Laptops with the EDAS software installed are required for this course. Installation instructions can be found online (at https://www.nccs.nasa.gov /services/analytics) and should be completed before the participants arrive at the conference, but help will be available on site as needed. The software has been tested on Linux Ubuntu 16.04 and above, Mac OSX 10.12.6 and above, and Windows 10 running the Windows subsystem for Linux. Instructions for installing Linux on Windows 10 can be found online (at https://docs .microsoft.com/en-us/windows/wsl /install-win10). Internet access will be provided. This short course is sponsored by the AMS Committee on Climate Variability and Change.

For more information, please contact Jerry Potter (email: gerald .potter@nasa.gov), Laura Carriere (email: laura.carriere@nasa.gov),Dan Duffy (email: daniel.q.duffy@nasa .gov), or Tom Maxwell (email: thomas .maxwell@nasa.gov).(10/18)

\section{ANNOUNCEMENT}

AMS Short Course on Introducing the Community Earth System Model, Version 2 (CESM2) via an Interactive Hands-On Tutorial Using the Cloud, 6 January 2019, Phoenix, Arizona

The Community Earth System Model (CESM) version 2, released in 2018, is the next generation, state-of-the-art Earth system model developed by the National Center for Atmospheric Research in collaboration with the university community. CESM can be used for prediction and understanding of a wide range of aspects of the Earth system on a variety of time scales. Moreover, CESM is freely available for use by the climate research community, making it an ideal tool for Earth system research and education. The capabilities within CESM range from idealized models of the atmosphere to comprehensive coupled simulations for leading edge research in Earth system science.

The goal of this short course is to introduce new users to CESM2. A broad overview of the capabilities within CESM2 will be provided along with hands-on experience at setting up and running simulations with CESM2.

This will be achieved through a half-day course consisting of an approximately 1-hour introductory lecture to CESM2 and the science that can be done with it, with the remaining time devoted to an interactive tutorial in which participants will gain experience at setting up and executing model simulations as well as making simple modifications to default configurations. The practical component of the tutorial will be performed on the Amazon Web Service (AWS) cloud.

By the end of this course, attendees will have the basic building blocks to start their own research with CESM2. They will gain a broad understanding of the various components of CESM2 and will become familiar with the framework used to run the model. Participants will also be provided with information on additional resources that can be used to further their familiarity with CESM2 and build on the basics learned during this short course. The course is aimed at new or potential users of
CESM2 who may be considering using CESM2 for their research or educational purposes. Each participant is required to come with their own laptop, capable of accessing wireless internet.

The instructors for the course are Cecile Hannay and Isla Simpson (Climate and Global Dynamics Laboratory of the National Center for Atmospheric Research). For more information please contact either Isla Simpson (email: islas@ucar.edu; tel.: 303-497-1763) or Cecile Hannay (email: hannay@ucar.edu; tel.: 303497-1327). (10/18)

\section{ANNOUNCEMENT}

\section{AMS Workshop on Diversity, Equity and Inclusion for Geoscientists, 6 January 2019, Phoenix, Arizona}

In 2018, the University Corporation for Atmospheric Research (UCAR) developed a 1-day DEI training for scientists, based on a highly successful 4-month UCAR/NCAR Equity \& Inclusion (UNEION) program. The one-day workshop, called UCAR/ NCAR Inclusion Training Experience (UNITE), aims to help people from all backgrounds explore their identities, ways in which power differentials operate across identity and professional roles, aspects of gender in the workplace, and how race and ethnicity are socialized and affect our experiences. The final hour of the workshop will be invested in exploring effective ways to broaden competencies around DEI at AMS and bring these ideas to others in the weather, water, and climate science field.

No prior experience or training in DEI discussions is expected, but participants will be required to invest around two hours of reading beforehand in order to ensure that everyone starts on the same page. The workshop is designed to enable participants from a range of levels and 
backgrounds and prior experiences to fully participate, as it largely focuses on understanding one's own identity and ways in which this can affects interactions at one's workplace. The only requirements for participation are an open mind: a willingness to be challenged and consider alternative viewpoints. For those with marginalized identities, please be aware that this is a learning space. While we will do everything we can, we cannot guarantee a completely safe space in this environment as others are learning and may microaggress during that process.

The course format consists of groups discussions and hands-on exercises that help to deepen participants' understanding of the topics covered. The instructors for this course will be Dr. Carolyn Brinkworth, Kristen Luna Aponte, Joshua Young, and Dr. Julie Malmberg (UCAR, Boulder, Colorado).

A lunch will be provided during the short course. Computers, laptops, or internet access will not be made available or required for this course. This workshop is co-sponsored by the AMS Board on Women and Minorities and UCAR. For more information please contact Carolyn Brinkworth (tel.: 303-497-1670; email: carolyn @ucar.edu). (10/18)
CALL FOR PAPERS

39th International Conference on Radar Meteorology, 16-20 September 2019, Nara Kasugano International Forum, Nara, Japan

The theme of the 39th Conference on Radar Meteorology is "Going to a new stage and new phase: Novel atmospheric sciences enabled by new technologies." The AMS Committee on Radar Meteorology and the local organizing committee in Japan welcome academics from a broad range of disciplines, including radar technologies, numerical modeling, satellite remote sensing, hydrology, signal processing, and atmospheric sciences on any aspect related to radar meteorology.

Abstracts are solicited on the following topics:

- spaceborne radar and its observation;

- phased array radar technology and its observation;

- wind profiler, cloud radar, and non-hydrometeorological radar;

- new and emerging radar technology;

- radar networks, quality control, processing, and software;

- quantitative precipitation estimation and hydrology;
- microphysical studies with radars;

- organized convection and severe phenomena; and

- use of radar data for nowcasting and numerical models

Important dates are as follows:

- website open: early 2019;

- abstract (1,000 words) due: 20 May 2019;

- notification of acceptance: early July 2019;

- program available: early July 2019;

- early registration due date: $1 \mathrm{Au}-$ gust 2019;

- extended abstract (optional and less than $10 \mathrm{MB}$ ) due date: $16 \mathrm{Au}$ gust 2019.

The best student oral presentation and poster presentation will be awarded the Spiros G. Geotis Prize. Students need to indicate their intent to participate in this competition during submission of their abstract.

For additional information please send an email to AMS_ICRM _LOC@ml.nict.go.jp. The local organizing committee is being chaired by Toshio Iguchi along with co-chairs Tomoo Ushio, Nobuhiro Takahashi, and Koyuru Iwanami and secretary Eiichi Yoshikawa. (10/18) 


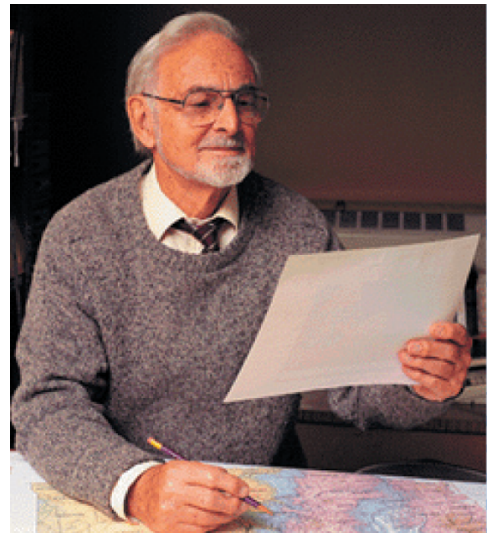

A Half Century of Progress A Meteorology:

edited by Richard H. Johnson and Robert A. Houze Jr.

with selections by: Lance F. Bosart Robert W. Burpee Anthony Hollingsworth James R. Holton Brian J. Hoskins Richard S. Lindzen John S. Perry Erik A. Rasmussen

Adrian Simmons Pedro Viterbo

A HALF CENTURY OF PROGRESS IN

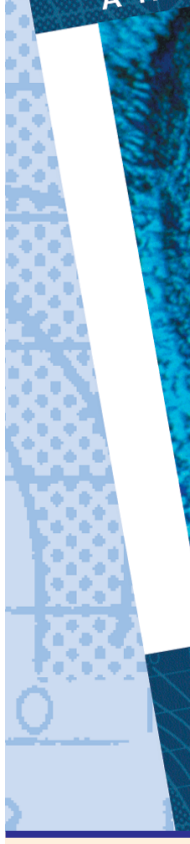

A

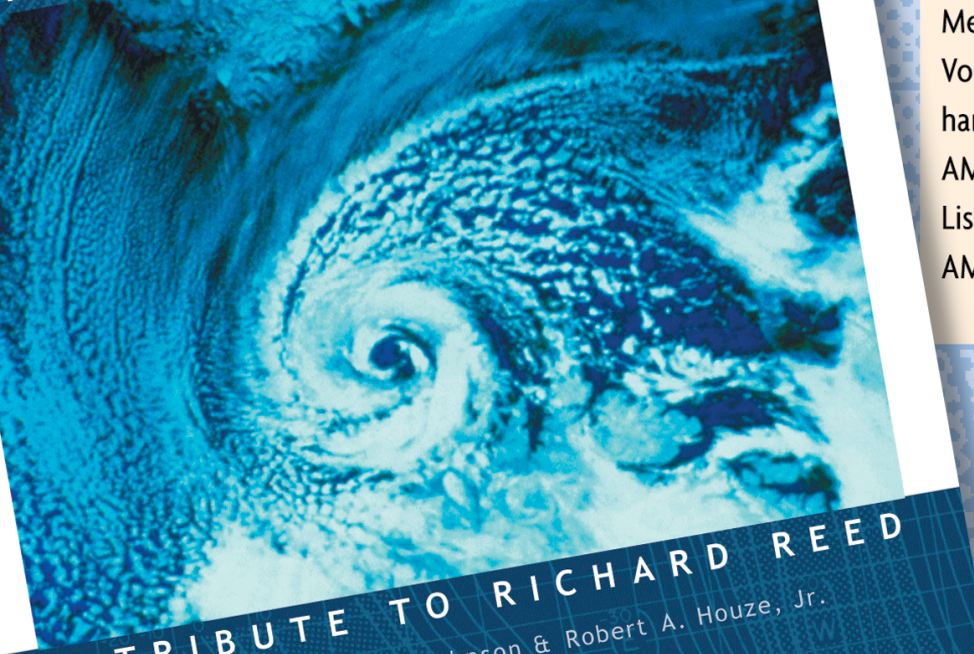

Through a series of reviews by invited experts, this monograph pays tribute to Richard Reed's remarkable contributions to meteorology and his leadership in the science community over the past 50 years. 2003.

Meterological Monograph Series, Volume 31, Number 53; 139 pages, hardbound; ISBN 1-878220-58-6; AMS Code MM53. List price: $\$ 80.00$ AMS Member price: $\$ 60.00$ 